Proceedings of the ISCIE International Symposium on Stochastic Systems Theory and Its Applications Osaka, Nov. 10-12, 1993

\title{
Time Moments for Continuous System Identification
}

\author{
AVB Subrahmanyam; DC Saha, and GP Rao \\ Department of Electrical Engineering, \\ Indian Institute of Technology, KHARAGPUR - 721 302, India
}

\begin{abstract}
Despite the popularity of rational transfer function matrix models for continuous-time MIMO systems, associated problems like "bias" due to measurement noise with equation error minimization, and reducibility of the estimated models, prompt for alternatives. This paper considers the use of models parametrized in terms of time moments of impulse response of continuous linear finite-dimensional MIMO systems. A novel algorithm presented in this paper gives bias-free irreducible estimates. Relevant implementation issues are discussed and simulation results are presented.
\end{abstract}

Keywords: System identification, parameter estimation, continuous-time systems, time moments.

\section{Introduction}

The use of finite-dimensional rational transfer function matrices (TFM) as identification models has been very popular for a parametric identification of continuous systems and several approaches were developed based on these models $[1,2]$. A majority of these approaches are based on minimization of the so-called equation-error (EE) by the least-squares (LS) technique. However, this minimization has a disadvantage that the parameter estimates are biased when the EE is not white. Another disadvantage is that such a formulation necessitates a canonical model having least common denominator (CD) for all the elements of the TFM. Due to this CD structure, the set of parameters is generally inflated and the estimates are reducible.

The present work aims at tackling these problems and a new approach towards irreducible TFM model estimation for CT MIMO systems operating in open-loop from sampled input-output measurements, is proposed. A new class of continuous models parametrized in terms of time moments of the system impulse response are proposed for this purpose. The proposed models are basically infinite dimensional, use of which is not practicable. Truncation of such models is unavoidable and to eliminate the resulting undermodelling error, an algorithm, in which the bias/error in the estimate is reparametrized in an iterative fashion, is proposed.

The layout of this paper is as follows.

Section 2 presents the description of the identification model. Section 3 presents an algorithm for removal of undermodelling error in parameter estimates. Relevant implementation issues are also discussed in this section. Results of numerical simulation experiments are presented in Section 4 and useful conclusions are drawn in Section 5 .

\section{Modelling via Time Moments}

Consider a stable linear time-invariant CT MIMO system with $\nu_{i}$ inputs and $\nu_{o}$ outputs modelled by the TFM

$$
\mathrm{G}(s)=\left[\begin{array}{cccc}
G_{11}(s) & G_{12}(s) & \cdots & G_{1 \nu_{i}}(s) \\
G_{21}(s) & G_{22}(s) & \cdots & G_{2 \nu_{i}}(s) \\
\vdots & \vdots & & \vdots \\
G_{\nu_{o} 1}(s) & G_{\nu_{o} 2}(s) & \cdots & G_{\nu_{o} \nu_{i}}(s)
\end{array}\right]_{\nu_{o} \times \nu_{i}}
$$

"E-mail: avbs@ee.iitkgp.ernet.in

${ }^{\dagger}$ Presently with the Engineering Systems Division, P \& D Plants, WED, PO Box 25862, ABU DHABI, UAE. 
where

$$
\begin{gathered}
G_{i j}(s)=\frac{B_{i j}(s)}{A_{i j}(s)}, \\
B_{i j}(s)=b_{o, i j}+b_{1, i j} s+\cdots+b_{n_{i j}-1, i j} s^{n_{i j}-1},
\end{gathered}
$$

and

$$
A_{i j}(s)=1+a_{1, i j} s+\cdots+a_{n_{i j}, i j} s^{n_{i j}} .
$$

Here " $s$ " denotes the Laplace operator and $n_{i j}$ is the order of the $i j$-th subsystem of $\mathrm{G}(\mathrm{s})$.

Let $\mathbf{u}(t)$ be the input signal, $\mathbf{x}(t)$ be the output signal and $\mathbf{y}(t)$ be the observation signal. Then we have the system input-output description

$$
\mathbf{X}(s)=\mathbf{G}(s) \mathbf{U}(s)
$$

where $\mathbf{X}(\mathrm{s})=\mathcal{L}[\mathbf{x}(\mathrm{t})]$ and $\mathrm{U}(\mathrm{s})=\mathcal{L}[\mathrm{u}(\mathrm{t})]$, " $\mathcal{L}$ " denoting the Laplace transformation.

Now, given the impulse response $T(t)$,

$$
\mathbf{T}(t)=\mathcal{L}^{-1}[\mathrm{G}(s)]
$$

the TFM G(s) may be expressed as

$$
\begin{aligned}
\mathbf{G}(s) & =\int_{0}^{\infty} \mathbf{T}(t) e^{-s t} d t \\
& =\sum_{l=0}^{\infty} \frac{(-1)^{l}}{l !} \mathbf{C}_{l} s^{l}
\end{aligned}
$$

where

$$
\mathbf{C}_{l}=\int_{0}^{\infty} t^{l} \mathbf{T}(t) d t \mathbf{C}_{l}, l=0,1,2, \ldots
$$

are the unnormalized time moments of $T(t)$. Note that $\left\{\mathrm{C}_{l}\right\}$ have dimension $\nu_{o} \times \nu_{i}$. It is more convenient to work with normalized time moments $\left\{\mathbf{M}_{l}\right\}$,

$$
\mathbf{M}_{l}=\frac{(-1)^{l}}{l !} \mathbf{C}_{l}
$$

Therefore

$$
\mathbf{G}(s)=\sum_{l=0}^{\infty} \mathbf{M}_{l} s^{l} .
$$

Denote

$$
\mathbf{M}_{l}=\left[\begin{array}{cccc}
m_{l, 11} & m_{l, 12} & \cdots & m_{l, 1 \nu_{i}} \\
m_{l, 21} & m_{l, 22} & \cdots & m_{l, 2 \nu_{i}} \\
\vdots & \vdots & & \vdots \\
m_{l, \nu_{o} 1} & m_{l, \nu_{o} 2} & \cdots & m_{l, \nu_{o} \nu_{i}}
\end{array}\right]
$$

Time moments of $i j$-th subsystem of $\mathrm{G}(\mathrm{s})$ are the corresponding entries of $\left\{\mathrm{M}_{l}\right\}$. These are further related to the coefficients of $A_{i j}(s)$ and $B_{i j}(s)$ as

$$
b_{r, i j}=m_{r, i j}+\sum_{h=0}^{r-1} m_{h, i j} a_{r-h, i j}, r=0, \ldots, n_{i j}-1
$$

and

$$
\left[\begin{array}{cccc}
m_{n_{i j}-1, i j} & m_{n_{i j}-2, i j} & \cdots & m_{0, i j} \\
\vdots & & & \\
m_{2 n_{i j}-2, i j} & m_{2 n_{i j}-3, i j} & \cdots & m_{n_{i j}-1, i j}
\end{array}\right]\left[\begin{array}{c}
a_{1, i j} \\
\vdots \\
a_{n_{i j}, i j}
\end{array}\right]=-\left[\begin{array}{c}
m_{n_{i j, i j}} \\
\vdots \\
m_{2 n_{i j}-1, i j}
\end{array}\right] .
$$

Now, in terms of $\left\{\mathbf{M}_{l}\right\}$, the system input-output relation may be expressed as

$$
\mathbf{x}(t)=\sum_{l=0}^{\infty} \mathbf{M}_{l} \mathbf{u}^{(l)}(t)
$$

where $(.)^{(l)}$ is the $l$-th time derivative of $($.$) .$ 


\subsection{The Model}

Considering only the first $(d+1)$ time moments, where $d$ is a positive integer, and neglecting the rest, we write the moment-based model as

$$
\tilde{\mathbf{y}}(t)=\sum_{l=0}^{d} \mathbf{M}_{l} \mathbf{u}^{(l)}(t) .
$$

Without loss of generality, the above model may be decomposed into $\nu_{o}$ number of equivalent MISO submodels for the row subsystems of the TFM. Thus consider the $i$-th row of equation (11), and dropping the subscript $i$ in all relevant symbols,

$$
\tilde{y}(t)=\sum_{l=0}^{d} \sum_{j=1}^{\nu_{i}} m_{l, j} u_{j}^{(l)}(t)
$$

or, with $p$ denoting the $\frac{d}{d t}$ operator,

$$
\tilde{y}(t)=\sum_{l=0}^{d} \sum_{j=1}^{\nu_{i}} m_{l, j} p^{l} u_{j}(t) .
$$

To avoid use of derivatives, the above equation is multiplied on both sides by a $(d+1)$-th order Poisson filter [3] $\frac{\beta^{d+1}}{(p+\lambda)^{d+1}}$, where $\lambda$ and $\beta$ are real positive constants. Therefore, denoting

$$
F_{l, d+1}(p)=\beta^{d+1} \frac{p^{l}}{(p+\lambda)^{d+1}}, l=0,1, \ldots, d
$$

equation (12) may be written as

$$
\mathcal{F}_{0, d+1}(p) \tilde{y}(t)=\sum_{l=0}^{d} \sum_{j=1}^{\nu_{i}} m_{l, i j} \mathcal{F}_{l, d+1}(p) u_{j}(t) .
$$

Thus we have the identification model

$$
\tilde{y}^{*}(t)=\Phi(t)^{T} \Theta
$$

where

where $\Phi_{j}(t)$,

$$
\begin{gathered}
\tilde{y}^{*}(t)=\mathcal{F}_{0, d+1}(p) \tilde{y}(t) \\
\Phi(t)=\left[\begin{array}{lll}
\Phi_{1}(t) & \cdots & \Phi_{\nu_{i}}(t)
\end{array}\right]^{T}
\end{gathered}
$$

$$
\Phi_{j}(t)=\left[\mathcal{F}_{0, d+1}(p) u_{j}(t) \cdots \mathcal{F}_{d, d+1}(p) u_{j}(t)\right]^{T}, j=1, \ldots, \nu_{i}
$$

and

$$
\Theta=\left[m_{0,1} \cdots m_{d, 1}|\cdots| m_{0, \nu_{i}} \cdots m_{d, \nu_{i}}\right]^{T} .
$$

Note that here $d \geq 2 n-1$, where $n=\max _{j}\left\{n_{j}\right\}$. Such a $d$ enables one to obtain coefficients of numerator and denominator polynomials of the entries of TFM. In general, considering $d_{j}$ time moments of the $j$-th subsystem of the MISO system under consideration, with $d_{j} \geq 2 n_{j}, \Phi(t)$ and $\Theta$ may be written as

$$
\Phi(t)=\left[\begin{array}{llll}
\Phi_{1}(t) & \cdots & \Phi_{\nu_{i}}(t)
\end{array}\right]^{T}
$$

where $\Phi_{j}(t), j=1, \ldots, \nu_{i}$

and

$$
\Phi_{j}(t)=\left[\begin{array}{llll}
\widetilde{r}_{0, d_{0}+1} & (p) u_{j}(t) \cdots \bar{F}_{d_{j}, d_{0}+1}(p) u_{j}(t)
\end{array}\right]^{T}
$$

where

$$
\Theta=\left[\begin{array}{lllllll}
m_{0,1} & \cdots & m_{d_{1}, 1}|\cdots| & m_{0, \nu_{i}} & \cdots & m_{d_{\nu_{i}}, \nu_{i}}
\end{array}\right]^{T}
$$

$$
d_{0}=\max _{j}\left\{d_{j}\right\} .
$$

In the above parameter estimation equation, note the virtual decoupling into non-interacting single-input/single output (SISO) elements. 


\subsection{Digital implementation and parameter estimation}

For the sake of implementation on digital computers, the differential operator $p$ and time $t$ are discretized via block-pulse functions, where

$$
p^{-1} f(t)=\frac{T}{2}\left(\frac{1+q^{-1}}{1-q^{-1}}\right) f(k)
$$

where $f(k)$ denotes the block-pulse value of $f(t)$ at $t=k T$ over the interval $[\overline{k-1} T, k T), T$ denoting the block-pulse width, and $q^{-1}$ denoting the usual backward shift operator. Thus

$$
\mathcal{F}_{l, d_{0}+1}\left(q^{-1}\right)=2^{l} T^{d_{0}+1-l} \frac{\left(1-q^{-1}\right)^{l}\left(1+q^{-1}\right)^{d_{0}+1-l}}{\left[(\lambda T+2)+(\lambda T-2) q^{-1}\right]^{d_{0}+1}} .
$$

Therefore the parameter estimation equation (equation (13)) is

$$
\tilde{y}^{*}(k)=\Phi(k)^{T} \Theta
$$

where

$$
\Phi(k)=\left[\Phi_{1}(k)^{T} \cdots \Phi_{\nu_{i}}(k)^{T}\right]^{T}
$$

where

$$
\Phi_{j}(k)=\left[\mathcal{F}_{0, d_{0}+1}\left(q^{-1}\right) u_{j}(k) \cdots \mathcal{F}_{d_{j}, d_{0}+1}\left(q^{-1}\right) u_{j}(k)\right]^{T}, j=1, \ldots, \nu_{i}
$$

Parameters of the above equation may be estimated using the simple recursive least-squares algorithm. In the subsequent sections we refer to this recursive algorithm as $\gamma\{\mathbf{u}(t), y(t), \Theta\}$ over $t \in\left[t_{0}, t_{N}\right]$.

\section{Bias reparametrization for TFM estimation}

The moment sequence is exactly finite when all the subsystems of $\mathrm{G}(s)$ are denominator free (a rare possibility !!). In such a case, the length of the moment sequence becomes $\max _{j}\left\{n_{j}\right\}$ and modelling via equation (11) becomes exact for any $d_{0} \geq \max _{j}\left\{n_{j}\right\}$. This situation can be met by adding fictitious zeros to each subsystem, to cancel all poles of the corresponding subsystem. In an identification experiment this is achievable by estimating denominators of subsystems and then cancelling them in an iterative fashion, to improve the quality of estimates. To illustrate, consider the case of $\nu_{i}=\nu_{0}=1$ such that

$$
X(s)=\frac{B(s)}{A(s)} U(s)
$$

For instance, if the denominator $A(s)$ is known exactly, we can write

$$
X(s)=\frac{B(s)}{A(s)} U(s)=\sum_{i=0}^{n-1} b_{i} s^{i} \bar{U}(s)
$$

where $\bar{U}(s)=\frac{1}{A(s)} U(s)$. Thus the model between $\bar{u}(t)$ and $y(t)$ has a finite moment sequence. This leads to the idea of "bias reparametrization" for bias elimination.

Now let $\frac{\hat{B}_{1}(s)}{\hat{A}_{1}(s)}$ be the initial estimate of $\frac{B(s)}{A(s)}$, which may be obtained using a truncated model as described in the previous section. Then

$$
\begin{aligned}
\frac{X(s)}{\bar{U}(s)} & =\frac{B(s)}{A(s)} \hat{A}_{1}(s)=\hat{A}_{1}(s) \Delta_{1}(s)+\hat{B}_{1}(s) \\
& =G_{1}(s)(\text { say } \ldots)
\end{aligned}
$$

where

$$
\Delta_{1}(s)=\frac{B(s)}{A(s)}-\frac{\hat{B}_{1}(s)}{\hat{A}_{1}(s)}
$$


is a measure of the bias. Note that the reference system is changed from $\frac{B(s)}{A(s)}$ to $\left\{\hat{A}_{1}(s) \Delta_{1}(s)+\hat{B}_{1}(s)\right\}$ which is a function of the bias measure $\Delta_{1}(s)$. When $\Delta_{1}(s)$ becomes zero $(\forall s)$, then and then only the reference system becomes denominator free.

Let $\frac{\hat{B}_{2}(s)}{\Delta \hat{A}_{1}(s)}$ be the estimate of $G_{1}(s)$. Therefore we may update

$$
\bar{u}(s)=\frac{1}{\Delta \hat{A}_{1}(s)} \frac{1}{\hat{A}_{1}(s)}=\frac{1}{\hat{A}_{2}(s)} u(s)
$$

and the new estimate becomes $\frac{\hat{B}_{2}(s)}{\hat{A}_{2}(s)}$. This procedure may be repeated till the moment sequence gets finitized.

We have the following "iterative" but "time-recursive" algorithm for the MISO subproblem:

\section{Algorithm 3.1 The algorithm has the following steps.}

1. Set iteration count $\xi=1$ and initialize $\hat{A}_{\xi, j}(p)=1.0, j=1, \ldots, \nu_{i}$.

2. Estimate $\hat{\Theta}_{\xi}$ using

$$
\gamma\left\{\left[\frac{1}{\hat{A}_{\xi, j}(p)} u_{j}(t), j=1, \ldots, \nu_{i}\right], y(t), \widehat{\Theta}_{\xi}\right\}
$$

over $t \in\left[t_{\xi-1}, t_{\xi}\right]$.

3. Calculate $\left\{\hat{a}_{i, j} ; i=1, \ldots, n_{j} ; j=1, \ldots, \nu_{i}\right\}$ from equation (9). Form $\Delta \hat{A}_{\xi, j}(p)=1+\hat{a}_{1, j} p+\cdots+\hat{a}_{n_{j}, j} p^{n_{j}}, j=$ $1, \ldots, \nu_{i}$.

4. Update $\hat{A}_{\xi+1, j}(p)=\hat{A}_{\xi, j}(p) \Delta \hat{A}_{\xi, j}(p) ; j=1, \ldots, \nu_{i}$.

5. Check for a finitized moment sequence of each subsystem. If so goto step 6 else set $\xi=\xi+1$ and goto step 2 .

6. Stop.

\subsection{Implementation issues}

1. The finite-dimensional time moment-based model (equation (11)) is valid only when the infinite series of $\mathrm{G}(s)$ defined in equation (7) is absolutely convergent. This is guaranteed when the upper limit of the sequence

$$
\left|m_{1}\right|,\left|m_{2}\right|^{\frac{1}{2}}, \ldots\left|m_{i}\right|^{\frac{1}{i}}, \ldots,
$$

$l=\varlimsup_{\lim _{i \rightarrow \infty}}\left|m_{i}\right|^{\frac{1}{i}}$ is equal to zero. To ensure this, the complex $s$-plane is expanded by a factor $\beta_{e}(>1)$, called as "band expansion factor," such that

$$
l=\varlimsup_{i \rightarrow \infty}\left(\frac{\left|m_{i}\right|}{\beta_{e}^{i}}\right)^{\frac{1}{i}}=0
$$

and thus absolute convergence is assured. It can be verified that the moment sequence is absolutely convergent when all the poles lie in the outside of the unit circle centered at the origin of the s-plane, and in such cases "band expansion" is not necessary. However, one has to rely on the a priori knowledge of the system poles, for this.

2. In the iterative process, order of $\hat{A}_{\xi+1, j}(p)$ increases linearly with $\xi$. To stop such an increase, we use the well-known moment-matching technique of model reduction [4] where the first $2 n_{j}$ moments of $\frac{1}{\widehat{A}_{\xi, j}(p) \Delta \hat{A}_{\xi, j}(p)}$ are matched to calculate an $n_{j}$-th order $\frac{1}{\hat{A}_{\xi+1, j}(p)}$. 
3. Since initial conditions affect the quality of estimates, they are to be estimated. Even if the system initial conditions are zero, the iterative modification of input signal on-line, makes the initial conditions (here "initial" means the starting time of any iteration) prominent. For the purpose of estimating the initial conditions, the data vector $\Phi(t)$ and the parameter vector $\Theta$ are augmented by $\left[\begin{array}{lllll}I_{0}(t) & I_{1}(t) & \cdots & I_{d_{0}-1}\end{array}\right]^{T}$ and $\left[\begin{array}{llll}c_{0} & c_{1} & \cdots & c_{d_{0}-1}\end{array}\right]^{T}$ respectively, where

$$
I_{i}(t)=\sum_{h=0}^{i} \frac{t^{d_{0}-i+h}}{\left(d_{0}-i+h\right) !}{ }^{i} C_{h}(-\lambda)^{h}
$$

and $c_{0}, c_{1}, \ldots, c_{d_{0}-1}$ are the parameters corresponding to initial conditions.

\section{Simulation experiments and results}

Consider a 2-input, 1-output system:

$$
\mathbf{G}(s)=\left[\frac{b_{0,1}}{1+a_{1,1} s+a_{2,1}} \frac{b_{0,2}}{1+a_{1,2} s}\right]
$$

with $\Theta_{G}=\left[\begin{array}{lllll}b_{0,1} & a_{1,1} & a_{2,1} & b_{0,2} & a_{1,2}\end{array}\right]^{T}=\left[\begin{array}{lllll}0.5 & 1.5 & 0.5 & 0.5 & 0.5\end{array}\right]^{T}$. PRBS signals of low mutual correlation are chosen as inputs. For parameter estimation, $d_{1,1}=5, d_{1,2}=3, \lambda=1, \beta=1, \beta_{e}=1$ are taken. The following cases of noise $(v(k))$ contained in system output $(x(k))$ are considered:

(i) White noise, $v(k)=w(k)$

(ii) Coloured noise,

$$
v(k)=\frac{0.04762-0.04762 q^{-1}}{1.0-1.904762 q^{-1}} w(k)
$$

where $w(k)$ is zero mean white stationary sequence. The variance of $w(k)$ is adjusted to obtain the desired signal-to-noise ratio $(S N R)$, in both the cases.

Table 1 shows the iterative estimate of $\Theta_{G}$ with white noise of $S N R=10$, and the pattern of parameter convergence is shown in Figures 1 and 2, for subsystems 1 and 2 respectively. Table 2 shows similar results in the presence of coloured noise of $S N R=10$. Results of Monte Carlo simulation of 20 experiments performed at $S N R$ ranging from 10.0 to 1.0 for the two types of noise sequences are shown in Tables 3 and 4 respectively.

In all these experiments Potter's square root implementation of the recursive LS algorithm [5] is used instead of the conventional recursive LS algorithm.

Discussion :

Iterative finitization of the moment sequence is observed in all the cases. See Figure 1. As expected, the effect of this finitization is seen in $\widehat{\Theta}_{G}$. See tables 1 , and 2 .

Results of Monte Carlo simulation experiments reveal that the $R E N$ is remarkably small at all noise levels (ranging from a very low of $10 \%$ to a very high of $100 \%$ ), and the standard deviation of parameter estimates is insignificant. These results indicate good consistency properties of the proposed algorithm in cases of both white and coloured noises. Results at very high noise levels can be improved further by taking large samples of measurements.

\section{Conclusions}

A new approach towards consistent irreducible model estimation of CT MIMO systems from sampled data has been proposed. Basically, the approach is to estimate denominator polynomials of all subsytems and then cancel them via a suitable filtering of the input signal, in an iterative fashion. To conclude, we indicate the advantageous features of the proposed algorithm, below:

- Because of a virtual decomposition of the identification model for the row subsystems of the MISO model into noninteracting SISO blocks, there is no parameter set inflation. Since time moments are system invariants like Markov parameters, realization of irreducible models is possible. 
- Owing to the special structure of the model (which is in fact, an approximation to the output error model), consistent estimates are obtained.

- The proposed approach is suitable for identification of structural invariants also. For the system described in equation (1), this is equivalent to finding order of each subsystem. In view of the decoupled estimation model, the order of any subsystem may be found by testing the rank of the associated Hankel matrix formed from the estimated moments of that subsystem. For this purpose, it is advisable to estimate a sufficiently long sequence of time moments for the sake of accurate order determination.

However, a drawback with the proposed approach is that the behaviour of the algorithm is not known in the case of approximate/reduced order modelling. In such a case, one has to be content with whatever estimates are obtained at the end of the first iteration. Nonetheless, the proposed approach has its own plus points and can replace existing approaches towards consistent estimation CT MIMO models.

\section{References}

[1] Unbehauen H. and G.P. Rao, "Continuous-time approaches to system identification - a survey," Automatica, vol. 26 , no. 1 , pp. $23,35,1990$.

[2] Sinha N.K. and G.P. Rao, eds., Identification of Continuous - time Systems : Methodology and Computer Implementation. Netherlands: Kluwer Academic Publishers, 1991.

[3] Saha D.C. and G.P Rao, Identification of Continuous Systems - A Poisson Moment Functional Approach. Lecture Notes in Control and Information Sciences-56, Berlin: Springer-Verlag, 1983.

[4] Jamshidi M., Large - Scale Systems : Modelling and Control. Netherlands: North-Holland, 1983.

[5] Bierman G.J., Factorization Methods for Discrete Sequential Estimation. Newyork: Academic Press, 1977.

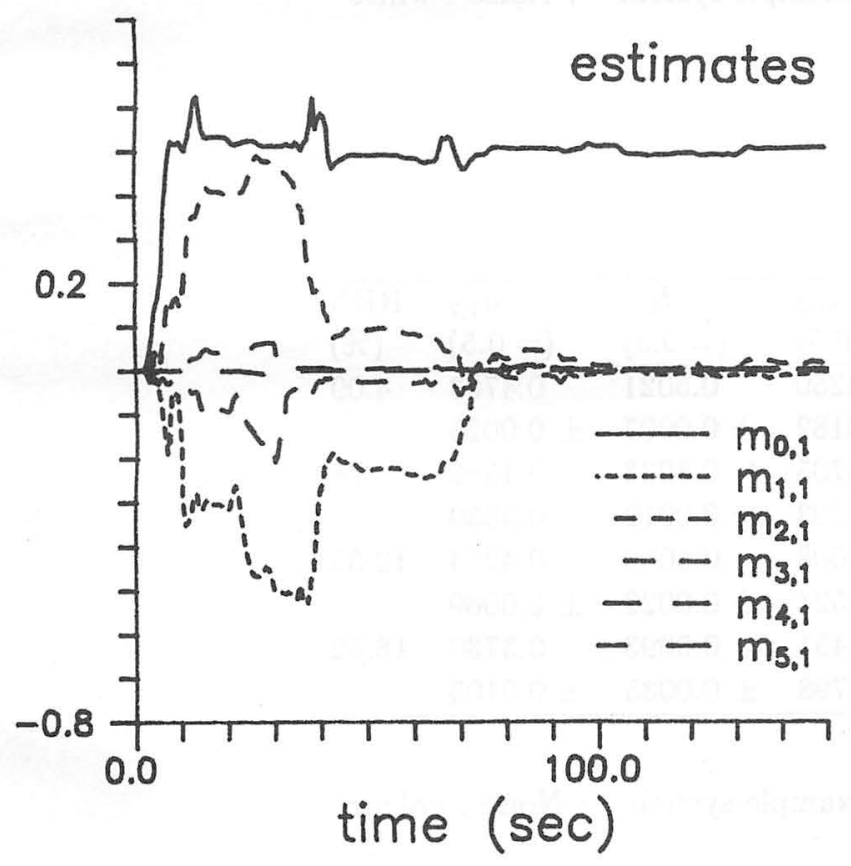

Figure 1: Pattern of parameter convergence - Subsystem 1, Noise: 10\% White

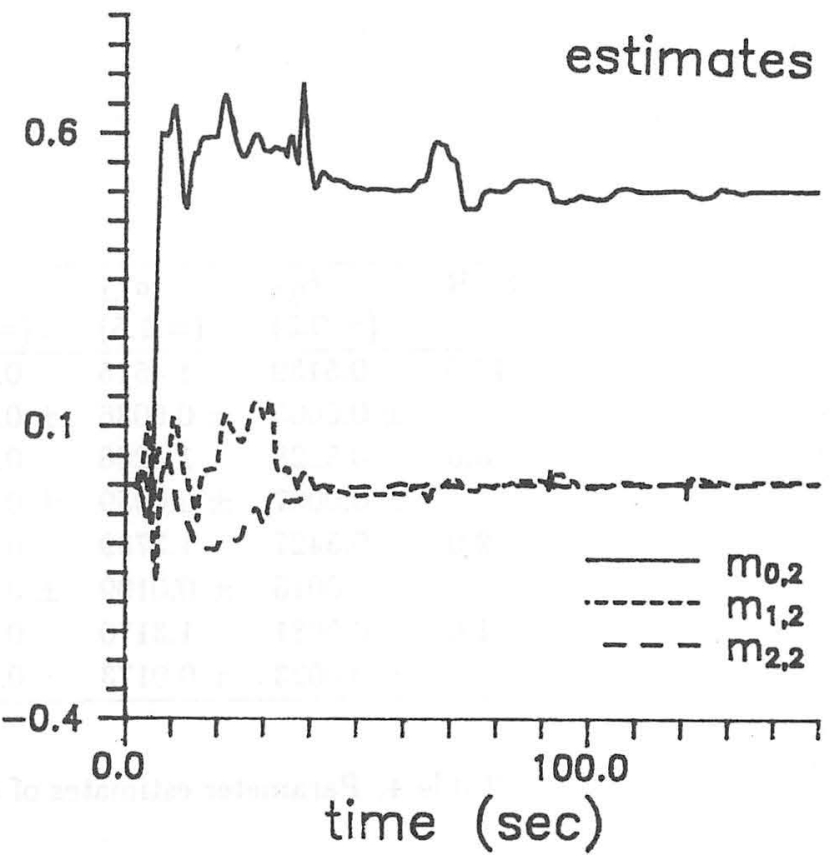

Figure 2: Pattern of parameter convergence - Subsystem 2, Noise: 10\% Colour 


\begin{tabular}{cccccc}
\hline Itr & $\begin{array}{c}b_{0,1} \\
\text { No }\end{array}$ & $\begin{array}{c}a_{1,1} \\
(=0.5)\end{array}$ & $\begin{array}{c}a_{1,2} \\
(=0.5)\end{array}$ & $\begin{array}{c}b_{0,2} \\
(=0.5)\end{array}$ & $\begin{array}{c}a_{1,2} \\
(=0.5)\end{array}$ \\
\hline 1 & 0.5130 & 0.9782 & 0.0467 & 0.5702 & 0.5807 \\
2 & 0.4837 & 1.4693 & 0.5979 & 0.5045 & 0.5215 \\
3 & 0.5048 & 1.4588 & 0.5024 & 0.5174 & 0.5219 \\
4 & 0.4950 & 1.4748 & 0.5186 & 0.5036 & 0.5048 \\
5 & 0.5067 & 1.4806 & 0.4934 & 0.5018 & 0.4846 \\
\hline
\end{tabular}

Table 1: Parameter estimates - Noise $=10 \%$ white

\begin{tabular}{cccccc}
\hline $\begin{array}{c}\text { Itr } \\
\text { No }\end{array}$ & $\begin{array}{c}b_{0,1} \\
(=0.5)\end{array}$ & $\begin{array}{c}a_{1,1} \\
(=1.5)\end{array}$ & $\begin{array}{c}a_{1,2} \\
(=0.5)\end{array}$ & $\begin{array}{c}b_{0,2} \\
(=0.5)\end{array}$ & $\begin{array}{c}a_{1,2} \\
(=0.5)\end{array}$ \\
\hline 1 & 0.5133 & 0.9752 & 0.0407 & 0.5701 & 0.5810 \\
2 & 0.4838 & 1.4701 & 0.5973 & 0.5045 & 0.5217 \\
3 & 0.5050 & 1.4586 & 0.5024 & 0.5177 & 0.5230 \\
4 & 0.4952 & 1.4751 & 0.5174 & 0.5037 & 0.5044 \\
5 & 0.5068 & 1.4806 & 0.4924 & 0.5018 & 0.4847 \\
\hline
\end{tabular}

Table 2: Parameter estimates - Noise $=10 \%$ colour

\begin{tabular}{rrrrrrr}
\hline SNR & $\begin{array}{r}b_{0,1} \\
(=0.5)\end{array}$ & $\begin{array}{r}a_{1,1} \\
(=1.5)\end{array}$ & $\begin{array}{r}a_{1,2} \\
(=0.5)\end{array}$ & $\begin{array}{r}b_{0,2} \\
(=0.5)\end{array}$ & $\begin{array}{r}a_{1,2} \\
(=0.5)\end{array}$ & $\begin{array}{r}\text { REN } \\
(\%)\end{array}$ \\
\hline 10.0 & 0.5068 & 1.4863 & 0.4967 & 0.5018 & 0.4875 & 0.88 \\
& \pm 0.0005 & \pm 0.0049 & \pm 0.0039 & \pm 0.0002 & \pm 0.0027 & \\
5.0 & 0.5109 & 1.4890 & 0.4844 & 0.5029 & 0.4818 & 1.25 \\
& \pm 0.0007 & \pm 0.0074 & \pm 0.0059 & \pm 0.0003 & \pm 0.0041 & \\
2.0 & 0.5201 & 1.4953 & 0.4567 & 0.5055 & 0.4693 & 2.49 \\
& \pm 0.0012 & \pm 0.0114 & \pm 0.0102 & \pm 0.0007 & \pm 0.0066 & \\
1.0 & 0.5320 & 1.5032 & 0.4221 & 0.5088 & 0.4537 & 4.21 \\
& \pm 0.0019 & \pm 0.0147 & \pm 0.0146 & \pm 0.0009 & \pm 0.0089 & \\
\hline
\end{tabular}

Table 3: Parameter estimates of example system : : Noise : white

\begin{tabular}{rrrrrrr}
\hline SNR & $\begin{array}{r}b_{0,1} \\
(=0.5)\end{array}$ & $\begin{array}{r}a_{1,1} \\
(=1.5)\end{array}$ & $\begin{array}{r}a_{1,2} \\
(=0.5)\end{array}$ & $\begin{array}{r}b_{0,2} \\
(=0.5)\end{array}$ & $\begin{array}{r}a_{1,2} \\
(=0.5)\end{array}$ & $\begin{array}{r}\text { REN } \\
(\%)\end{array}$ \\
\hline 10.0 & 0.5139 & 1.4515 & 0.4250 & 0.5021 & 0.4753 & 4.09 \\
& \pm 0.0005 & \pm 0.0036 & \pm 0.0182 & \pm 0.0007 & \pm 0.0025 & \\
5.0 & 0.5228 & 1.4286 & 0.3705 & 0.5033 & 0.4582 & 6.78 \\
& \pm 0.0007 & \pm 0.0059 & \pm 0.0293 & \pm 0.0012 & \pm 0.0039 & \\
2.0 & 0.5427 & 1.3789 & 0.2608 & 0.5059 & 0.4204 & 12.35 \\
& \pm 0.0015 & \pm 0.0109 & \pm 0.0524 & \pm 0.0022 & \pm 0.0069 & \\
1.0 & 0.5681 & 1.3170 & 0.1451 & 0.5093 & 0.3730 & 18.52 \\
& \pm 0.0023 & \pm 0.0173 & \pm 0.0798 & \pm 0.0035 & \pm 0.0105 & \\
\hline
\end{tabular}

Table 4: Parameter estimates of example system : Noise : colour 